\title{
Echo derived variables predicting exercise tolerance in patients with dilated and poorly functioning left ventricle
}

\author{
K M Webb-Peploe, M Y Henein, A J S Coats, D G Gibson
}

\begin{abstract}
Objective-To determine whether resting echo derived measurements predict exercise tolerance and its interrelation with heart rate response and ventilation drive in patients with systolic left ventricular disease.

Design-Prospective echocardiographic examination followed by cardiopulmonary exercise testing.

Setting-A tertiary referral centre for cardiac diseases.

Subjects-21 patients (11 with coronary artery disease, 10 with idiopathic dilated cardiomyopathy) with end diastolic dimension $>6.4 \mathrm{~cm}$, shortening fraction $<25 \%$, and in sinus rhythm. There were 11 age matched normal controls. Results-In the patients, peak oxygen consumption $\left(\mathbf{m} \dot{V}_{\mathrm{O}_{2}}\right)$ correlated with right ventricular long axis excursion $(r=0.62)$; $65 \%$ of the variance in $\mathbf{m V} \mathrm{o}_{2}$ was predictable using a multivariate model with right ventricular long axis excursion and peak lengthening rate, and peak mitral atrial filling velocity as independent variables. Aetiology was not an independent predictor, although the right ventricular long axis excursion (mean (SD)) was greater in patients with idiopathic dilated cardiomyopathy than in those with coronary artery disease $(2.4(0.5) \mathrm{cm} \quad v \quad 1.6(0.5) \mathrm{cm}$, $\mathrm{p}<0.001)$. Peak heart rate correlated with duration of mitral regurgitation $(r=-0.52)$ and the slope of ventilation against $\mathrm{CO}_{2}$ production correlated with $M$ mode isovolumic relaxation time $(r=0.61)$.

Conclusions-In patients with systolic left ventricular dysfunction, more than half the variance in exercise tolerance can be predicted by factors measured on echocardiography at rest, particularly right ventricular long axis excursion.

(Heart 1998;80:565-569)
\end{abstract}

Department of

Cardiology, Royal

Brompton Hospital,

Sydney Street, London

SW3 6NP, UK

K M Webb-Peploe

M Y Henein

A J S Coats

D G Gibson

Correspondence to:

Dr Gibson.

Accepted for publication 17 July 1998 ing exercise ( $\dot{\mathrm{V}} \mathrm{E} / \dot{\mathrm{V}}_{\mathrm{CO}}$ slope). Standard measures of cardiac function such as left ventricular ejection fraction and pulmonary capillary wedge pressure have not consistently been shown to predict exercise capacity in such patients. ${ }^{2-4}$ Resting right ventricular function as assessed by nuclear angiography, however, does appear to correlate more consistently with functional capacity in the presence of left ventricular impairment. ${ }^{56}$

Our aim in this study was not to investigate basic mechanisms but rather to determine the extent to which exercise tolerance and ventilation pattern could be predicted from resting non-invasive echo Doppler measurements in a group of patients with left ventricular dilatation and a wide range of functional impairment ( $\mathrm{mV} \mathrm{O}_{2}$ ranged from 13.0 to $31.6 \mathrm{ml} / \mathrm{kg} / \mathrm{min}$ ). Peak oxygen consumption and ventilation pattern are increasingly being used as surrogate markers of prognosis particularly in patients being assessed for heart transplantation. ${ }^{7}$ Serial resting echocardiography might prove a less stressful means of monitoring such patients' clinical progress than repeated cardiopulmonary exercise testing.

In this study, echocardiography and cardiopulmonary exercise testing were performed in a selected group of patients with left ventricular dilatation who were starting a trial of exercise training in heart failure. They were compared with age matched controls recruited for the same study.

\section{Methods}

All patients studied had a left ventricular end diastolic dimension greater than $6.4 \mathrm{~cm}$, a shortening fraction less than $25 \%$, and were in sinus rhythm. They were able to exercise on the treadmill to exhaustion, limited by fatigue or shortness of breath, and not by angina. None had obstructive lung disease as assessed by spirometry. They had been stable symptomatically on the same medications for at least two months before the tests. Eleven patients (mean (SD) age 55 (6) years) had coronary artery disease diagnosed on coronary angiography, and 10 (age 51 (4) years) had a normal coronary arteriogram and were considered to have idiopathic dilated cardiomyopathy. Five patients with coronary artery disease had had previous bypass surgery. All except one of the remainder had sustained a previous documented myocardial infarct. Other clinical details are summarised in table 1 . For the purposes of comparison, a daily dose of $1 \mathrm{mg}$ of bumetanide was taken as equivalent to $40 \mathrm{mg}$ 
Table 1 Clinical data

\begin{tabular}{llll}
\hline Variable & Controls & Ischaemic & Idiopathic \\
\hline Age (years) & $51(7)$ & $55(6)$ & $51(4)$ \\
Sex; male/female & $10 / 1$ & $11 / 0$ & $9 / 1$ \\
Equivalent frusemide dose (mg) & & $107(98)$ & $100(85)$ \\
Bendrofluazide (mg) & $0.9(1.7)$ & $1.3(2.1)$ \\
Equivalent enalapril dose (mg) & & $22(11)$ & $9(7)+\dagger$ \\
Patients on & & 1 \\
$\quad$ Long acting nitrates & 8 & 1 \\
$\quad$ Aspirin & 8 & 5 \\
$\quad$ Warfarin & 3 & 5 \\
$\quad$ Digoxin & 3 & \\
\hline
\end{tabular}

Values are mean $(\mathrm{SD}) ;+t \mathrm{p}<0.01 v$ ischaemic group.

of frusemide, and $75 \mathrm{mg}$ of captopril daily was taken as equivalent to $10 \mathrm{mg}$ of enalapril, $5 \mathrm{mg}$ of lisinopril, or $2.5 \mathrm{mg}$ of ramipril.

The 11 normal controls (age 51 (7) years) were volunteers who had no symptoms suggestive of heart disease and no history of hypertension, effort induced chest pain, or shortness of breath. Resting electrocardiograms were normal in all (one had right bundle branch block). There was no evidence of significant heart disease on echocardiography and no ischaemic electrocardiogram changes on maximal exercise testing.

All patients and controls gave written informed consent before starting the study, which was approved by the Royal Brompton Hospital ethics committee.

\section{ECHOCARDIOGRAPHY}

Patients were asked to omit the diuretic dose immediately before testing, but took their other drugs as usual. Echocardiography was performed at rest with the subject in the left lateral position, using a $2.5 \mathrm{MHz}$ imaging transducer connected to a Hewlett-Packard Sonos 1500 echocardiograph (Hewlett-Packard Inc, Andover, Massachusetts, USA). A cross sectional guided $M$ mode echocardiography of left ventricular short axis was performed from the parasternal long axis view with the cursor placed at the tip of the mitral valve leaflets. Long axis

Table 2 Echocardiographic measurements

\begin{tabular}{llll}
\hline Variable & Controls & Ischaemic & Idiopathic \\
\hline Left ventricular short axis & & & \\
End diastolic dimension $(\mathrm{cm})$ & $4.8(0.5)$ & $7.4(0.6)^{\star \star \star}$ & $7.1(0.6)^{\star \star \star}$ \\
End systolic dimension $(\mathrm{cm})$ & $3.0(0.6)$ & $6.4(0.6)^{\star \star \star}$ & $6.1(0.7)^{\star \star \star}$ \\
Fractional shortening $(\%)$ & $38(8)$ & $13(4)^{\star \star \star}$ & $14(5)^{\star \star \star}$ \\
A2-mitral cusp separation $(\mathrm{ms})$ & $60(20)$ & $40(40)$ & $60(45)$ \\
Peak shortening rate $(\mathrm{cm} / \mathrm{s})$ & $8.8(2.9)$ & $4.9(1.3)^{\star \star \star}$ & $4.5(2.1)^{\star \star \star}$ \\
Peak lengthening rate $(\mathrm{cm} / \mathrm{s})$ & $9.4(3.0)$ & $6.6(3.2)^{\star}$ & $6.1(2.8)^{\star}$ \\
Left ventricular long axis & & & \\
Lateral & & & $0.9(0.3)^{\star \star \star}$ \\
Excursion $(\mathrm{cm})$ & $1.5(0.3)$ & $0.9(0.3)^{\star \star \star}$ & $4.7(0.8)^{\star \star \star}$ \\
Peak shortening rate $(\mathrm{cm} / \mathrm{s})$ & $7.3(1.9)$ & $4.3(1.3)^{\star \star \star}$ & $5.4(3.0)^{\star}$ \\
Peak lengthening rate $(\mathrm{cm} / \mathrm{s})$ & $7.7(1.7)$ & $4.9(2.8)^{\star}$ & \\
Septal & & & $0.7(0.3)^{\star \star \star}$ \\
Excursion $(\mathrm{cm})$ & $1.4(0.3)$ & $0.6(0.3)^{\star \star \star}$ & $3.4(1.3)^{\star \star \star}$ \\
Peak shortening rate $(\mathrm{cm} / \mathrm{s})$ & $6.1(1.4)$ & $2.9(1.8)^{\star \star \star}$ & \\
Peak lengthening rate $(\mathrm{cm} / \mathrm{s})$ & $6.5(2.1)$ & $3.1(2.2)^{\star \star \star}$ & $4.7(1.8)$ \\
Posterior & & & \\
Excursion $(\mathrm{cm})$ & $1.6(0.3)$ & $0.9(0.2)^{\star \star \star}$ & $1.0(0.3)^{\star \star \star}$ \\
Peak shortening rate $(\mathrm{cm} / \mathrm{s})$ & $6.5(1.3)$ & $4.0(1.4)^{\star \star \star}$ & $4.0(1.7)^{\star \star \star}$ \\
Peak lengthening rate $(\mathrm{cm} / \mathrm{s})$ & $7.9(2.1)$ & $5.2(2.2)^{\star \star}$ & $4.5(2.2)^{\star \star}$ \\
Right ventricular long axis & & & \\
Excursion $(\mathrm{cm})$ & $2.6(0.3)$ & $1.6(0.5)^{\star \star \star}$ & $2.4(0.5) \dagger+\dagger$ \\
Peak shortening rate $(\mathrm{cm} / \mathrm{s})$ & $12.1(1.6)$ & $8.6(3.0)^{\star \star}$ & $11.3(3.5)^{\star \star}$ \\
Peak lengthening rate $(\mathrm{cm} / \mathrm{s})$ & $10.1(3.1)$ & $8.5(2.7)$ & $10.2(3.6)$ \\
\hline Val & &
\end{tabular}

Values are mean (SD). A2-mitral cusp separation, $M$ mode isovolumic relaxation time measured as the interval between the first high frequency component on the phonocardiogram and the separation of the mitral valve leaflets on the mitral $M$ mode echocardiogram.

${ }^{\star \star \star} \mathrm{p}<0.001,{ }^{\star \star} \mathrm{p}<0.01,{ }^{\star} \mathrm{p}<0.05 v$ controls; $+\dagger+\mathrm{p}<0.001,+\mathrm{p}<0.05 v$ ischaemic group. recordings were obtained from the apical four and two chamber views, with the cursor across three sites on the left atrioventricular ring (lateral, septal, and posterior), and across the tricuspid ring in the four chamber view, representing the right ventricular free wall, as previously described. ${ }^{8}$ At all sites, we measured total excursion of the atrioventricular ring together with peak shortening and lengthening rates. We also recorded forward transmitral Doppler flow velocities from the apical four chamber view, using the same transducer in pulsed wave Doppler mode, with the sample volume adjacent to the tips of leaflets. Any mitral regurgitation was recorded using a nonimaging continuous wave Doptek transducer (Doptek, London, UK). All $\mathrm{M}$ mode traces were measured and digitised as previously described. $^{8}{ }^{9}$

\section{EXERCISE TESTING}

Cardiopulmonary exercise testing was performed after the echocardiography. Each subject exercised to exhaustion on a treadmill using the standard Bruce protocol ${ }^{10}$ with the addition of "stage 0 " at $1.0 \mathrm{mph}$ and a 5\% gradient. Subjects breathed through a one way valve, allowing the collection of expired air. Expired air was mixed with an inert indicator gas in a mixing chamber, and samples taken every 11 seconds were analysed by mass spectrometry (Amis 2000, Innovision, Odense, Denmark). Ventilation rate ( $\dot{\mathrm{V}} \mathrm{E})$, carbon dioxide production $\left(\mathrm{V}_{\mathrm{CO}_{2}}\right)$, and oxygen consumption $\left(\dot{\mathrm{V}}_{2}\right)$ were derived on-line every 11 seconds. ${ }^{1}$ In each exercise test, we calculated peak oxygen consumption $\left(\mathrm{mV} \mathrm{O}_{2}\right)$, the $\dot{\mathrm{V}} \mathrm{E} /$ $\dot{\mathrm{V}}_{\mathrm{CO}_{2}}$ slope, and the respiratory exchange ratio- $\dot{\mathrm{V}} \mathrm{CO}_{2} / \dot{\mathrm{V}}_{2}$ (respiratory quotient, $\mathrm{RQ}$ ) at peak exercise.

\section{STATISTICAL ANALYSIS}

Continuous data are expressed as mean (SD). The data were analysed using Statview (Abacus Concepts Inc, Berkeley, California, USA). The differences between groups were compared by analysis of variance. When such differences were present, Fisher's test was used to determine which of the means differed significantly. When comparing only two groups (for example, drug dosage in the two patient groups) the Student's unpaired $t$ test was used. To analyse relations between variables, simple linear regression (least squares method), stepwise regression, and multivariate analysis were performed. Results were considered to be significant at $\mathrm{p}<0.05 . R^{2}$, or the proportion of the dependent variable's variability that is explained by the independent variables, was used to assess the quality of the regression models. "Adjusted $R^{2 \text { " }}$ adjusts for the number of variables in the model as $R^{2}$ is likely to increase simply by increasing the number of independent variables.

\section{Results}

\section{CLINICAL DATA}

The two patient groups with and without coronary artery disease did not differ significantly 
Table 3 Mitral Doppler echocardiography

\begin{tabular}{llll}
\hline Variable & Controls & Ischaemic & Idiopathic \\
\hline E wave velocity (m/s) & $0.6(0.2)$ & $0.8(0.5)$ & $0.5(0.4)$ \\
E wave deceleration time (ms) & $200(40)$ & $180(80)$ & $140(35)$ \\
A wave velocity (m/s) & $0.5(0.1)$ & $0.3(0.3)$ & $0.8(0.2)^{\star}+\dagger \dagger$ \\
E/(E+A) ratio & $0.6(0.1)$ & $0.7(0.4)$ & $0.4(0.2)+\dagger$ \\
A2-onset of flow (ms) & $95(10)$ & $90(30)$ & $120(20)^{\star}+\dagger$ \\
A2-peak of E wave (ms) & $175(20)$ & $155(40)$ & $200(45)$ \\
Duration of flow (ms) & $545(130)$ & $340(200)^{\star \star \star}$ & $345(145)^{\star \star}$ \\
Duration of regurgitation (ms) & $400(15)$ & $425(50)$ & $445(30)$ \\
\hline
\end{tabular}

Values are mean (SD). A2, first high frequency component of the second heart sound on the phonocardiogram; A2-onset of flow, the Doppler isovolumic relaxation time measured as the interval between A2 and the onset of flow across the mitral valve; A velocity, peak velocity of the atrial component of mitral filling; E velocity, peak velocity of the early component of mitral filling; $\mathrm{E} /(\mathrm{E}+\mathrm{A})$, ratio of peak early filling velocity to the sum of the peak early and the peak atrial filling velocities.

${ }^{\star \star \star} \mathrm{p}<0.001,{ }^{\star \star} \mathrm{p}<0.01,{ }^{\star} \mathrm{p}<0.05 v$ controls; $+\dagger+\mathrm{p}<0.001,+\dagger \mathrm{p}<0.01 v$ ischaemic group.

with respect to age or diuretic dose. Clinical data for patients and controls are summarised in table 1 .

ECHO DOPPLER

By definition, both patient groups had increased left ventricular end diastolic and end systolic dimensions with reduced fractional shortening, but they did not differ significantly from each other in standard measures of short and long axis left ventricular function (table 2). The Doppler data are summarised in table 3. All patients had a mitral filling time that was significantly shorter than in the control group. Six patients with coronary artery disease filled only during early diastole, two of each patient group filled solely during atrial systole, and three with coronary artery disease and eight with idiopathic dilated cardiomyopathy had early and late components to their filling pattern. Mild functional mitral regurgitation was detectable in all patients with coronary artery disease, in five with idiopathic dilated cardiomyopathy, and in two normal controls.

Mean right ventricular long axis excursion and peak shortening rate were reduced in patients with coronary artery disease (table 2). The ranges of right ventricular long axis excursion were 1.8 to $3.3 \mathrm{~cm}$ in patients with idiopathic dilated cardiomyopathy and 1.1 to $2.8 \mathrm{~cm}$ in patients with coronary artery disease; thus some of the patients with coronary artery disease had relatively preserved right ventricular function.

EXERCISE DATA

The exercise data for all groups are summarised in table 4 . The exercise capacity of the patients with idiopathic dilated cardiomyopathy was significantly greater than in those with coronary artery disease.

Table 4 Exercise data

\begin{tabular}{llll}
\hline Variable & Controls & Ischaemic & Idiopathic \\
\hline Exercise time (s) & $952(106)$ & $503(89)^{\star \star \star}$ & $722(117)^{\star \star \star}+\dagger+\dagger$ \\
$\mathrm{m} \dot{V} \mathrm{o}_{2}(\mathrm{ml} / \mathrm{kg} / \mathrm{min})$ & $31.5(4.6)$ & $18.6(3.9)^{\star \star \star}$ & $25.0(4.9)^{\star \star}+\dagger$ \\
$\dot{\mathrm{VE}} / \mathrm{V}^{\circ} \mathrm{CO}_{2}$ & $25.5(3.8)$ & $35.8(5.3)^{\star \star \star}$ & $31.1(4.7)^{\star} \dagger$ \\
Anaerobic threshold $(\mathrm{ml} / \mathrm{kg} / \mathrm{min})$ & $14.0(4.1)$ & $11.3(3.3)$ & $13.1(3.4)$ \\
$\mathrm{RQ}$ & $1.27(0.13)$ & $1.18(0.11)$ & $1.19(0.09)$ \\
Resting heart rate (beats/min) & $66(9)$ & $83(20)^{\star \star}$ & $88(10)^{\star \star}$ \\
Peak heart rate (beats/min) & $168(20)$ & $144(25)^{\star \star}$ & $167(12) \dagger$ \\
Change in heart rate (beats/min) & $102(15)$ & $61(12)^{\star \star \star}$ & $79(14)^{\star \star \star}+\dagger$ \\
\hline
\end{tabular}

Values are mean (SD). $m \dot{V}$, peak oxygen consumption; $\mathrm{RQ}$, ratio of carbon dioxide production to oxygen consumption at peak exercise; $\mathrm{VE} / \mathrm{VCO}_{2}$, regression slope of ventilation rate against carbon dioxide production during exercise.

${ }^{\star \star \star}{ }^{\star} \mathrm{p}<0.001,{ }^{\star \star} \mathrm{p}<0.01,{ }^{\star} \mathrm{p}<0.05 v$ controls; $+\dagger+\mathrm{p}<0.001,+\dagger \mathrm{p}<0.01,+\mathrm{p}<0.05 v$ ischaemic group.

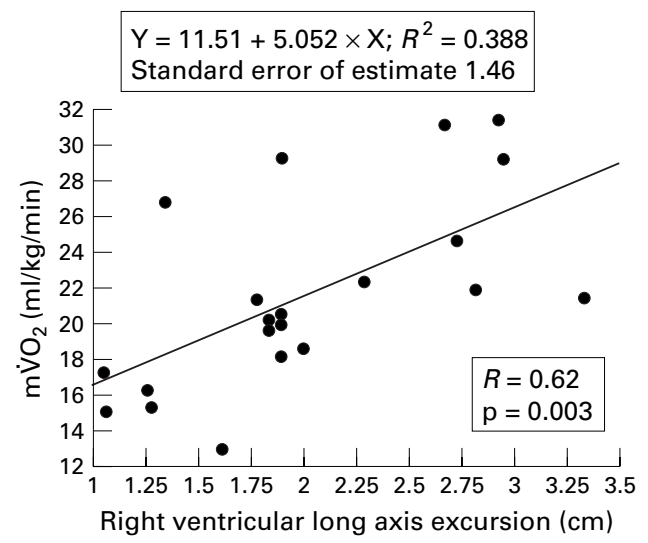

Figure 1 Relation between peak oxygen consumption $\left(m \dot{V}_{2}\right)$ and right ventricular long axis excursion in patients in whom the left ventricle is dilated and poorly functioning.

As would be expected, in the patients as a whole the peak oxygen consumption correlated with exercise time $(r=0.70)$, with anaerobic threshold $\quad(r=0.77)$, with $\quad \dot{\mathrm{V}} / \dot{\mathrm{V}}_{\mathrm{CO}_{2}}$ $(r=-0.55)$, and with pulse rate change $(r=0.54)$.

\section{CORRELATIONS BETWEEN ECHO DOPPLER AND}

\section{EXERCISE CAPACITY}

Predictors of peak oxygen consumption

The single variables that had the highest correlation with $\mathrm{mV}_{\mathrm{O}_{2}}$ were right ventricular long axis excursion $(r=0.62$ ) (fig 1$)$, peak mitral A velocity $(r=0.56)$, and septal long axis excursion $(r=0.47) \cdot \mathrm{mV}_{\mathrm{O}_{2}}$ was not correlated with either left ventricular lateral long axis excursion $(r=0.20)$ or left ventricular posterior long axis excursion $(r=0.02)$. Including aetiology with right ventricular long axis excursion in a multivariate model predicting $\mathrm{m} \dot{V}_{\mathrm{O}_{2}}$ did not cause a significant increase in $R$, the coefficient of multiple correlation. Peak oxygen consumption also showed no correlation with left ventricular short axis dimensions or shortening fraction. In a stepwise regression model entering all the measured echocardiographic variables for which the data were complete for every patient, right ventricular long axis excursion, mitral A wave velocity, and right ventricular long axis peak shortening rate emerged as the independent predictors of peak oxygen consumption (table 5). Entering these as independent variables in a multivariate model, $65 \%$ of the variance of peak oxygen consumption was predictable.

\section{Predictors of ventilatory drive during exercise}

The $\dot{\mathrm{VE}} / \dot{\mathrm{V}}_{\mathrm{CO}}$ slope correlated with $\mathrm{M}$ mode isovolumic relaxation time $(r=0.61)$ (fig 2). The scatter plot of the association shows that patients either fell into the normal range with a lower $\dot{\mathrm{VE}} / \dot{\mathrm{V}}_{\mathrm{CO}}$ slope that bore no relation to isovolumic relaxation time or into an abnormal group with a higher $\dot{\mathrm{V}} / \dot{\mathrm{V}}_{\mathrm{CO}_{2}}$ that correlated closely with isovolumic relaxation time. The shorter the isovolumic relaxation time, the higher the $\dot{\mathrm{V}} \mathrm{E} / \dot{\mathrm{V}} \mathrm{O}_{2}$ slope. 
Table 5 Stepwise and multiple regression analysis showing predictors of peak oxygen consumption

\begin{tabular}{|c|c|c|c|c|}
\hline & Coefficient & F to remove & $R$ & Adjusted $R^{2}$ \\
\hline \multicolumn{5}{|l|}{ Stepwise regression } \\
\hline Step 1 & & & 0.62 & 0.36 \\
\hline RV long axis excursion & 5.05 & 12.05 & & \\
\hline Step 2 & & & 0.77 & 0.56 \\
\hline RV long axis excursion & 11.18 & 23.03 & & \\
\hline RV long axis peak shortening rate & -1.39 & 9.48 & & \\
\hline Step 3 & & & 0.84 & 0.65 \\
\hline RV long axis excursion & 9.93 & 21.80 & & \\
\hline RV long axis peak shortening rate & -1.41 & 12.49 & & \\
\hline Mitral A wave velocity & 5.50 & 5.94 & & \\
\hline Intercept & 12.67 & & & \\
\hline Multiple regression & & $p$ Value & & \\
\hline RV long axis excursion & & 0.0002 & & \\
\hline RV long axis peak shortening rate & & 0.0025 & & \\
\hline Mitral A wave velocity & & 0.026 & & \\
\hline
\end{tabular}

Mitral A wave velocity, peak velocity of the atrial component of mitral filling; RV, right ventricular.

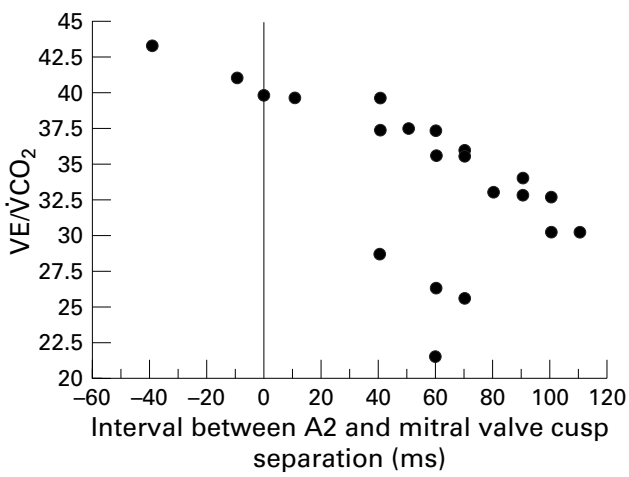

Figure 2 Relation between ventilation drive $\left(\dot{V}_{E} / \dot{V}_{\mathrm{CO}_{2}}\right)$ and $M$ mode isovolumic relaxation time in patients with a dilated and poorly functioning left ventricle.

Predictors of peak heart rate on exercise

Peak heart rate attained on exercise correlated inversely with peak $\mathrm{E}$ wave velocity $(r=-0.52)$, with $\mathrm{E} /(\mathrm{E}+\mathrm{A})$ ratio (the ratio of peak early filling velocity to the sum of the peak early and peak atrial filling velocities) $(r=-0.55)$, with duration of mitral regurgitation $(r=-0.52)$, and with age $(r=-0.55)$.

\section{Discussion}

Exercise tolerance is often reduced in patients with systolic left ventricular disease. Neither echo derived measures of left ventricular systolic function nor left ventricular ejection fraction determined by nuclear angiography predict exercise tolerance. ${ }^{2}$ Resting pulmonary artery wedge pressure has been shown both to correlate with ${ }^{11}$ and to have no influence on ${ }^{12}$ peak oxygen consumption and exercise capacity. Similarly, lower New York Heart Association functional class or better exercise tolerance have been linked to both low ${ }^{13}$ and high ${ }^{14} \mathrm{E} / \mathrm{A}$ ratios.

We confirmed that left ventricular cavity size and shortening fraction at rest do not predict exercise tolerance. Transmitral flow velocities showed some correlation in that a preserved A wave (that is, the absence of a restrictive filling pattern) was associated with greater exercise tolerance. Though the patients in this trial were indistinguishable in measures of left ventricular size and systolic function as assessed using standard $M$ mode echocardiography, those with coronary artery disease performed significantly less well than those with idiopathic dilated cardiomyopathy. In the final analysis, however, aetiology did not prove to be an independent predictor. The major factor predicting peak oxygen consumption was the extent of right ventricular long axis excursion, while septal long axis excursion and transmitral flow pattern were less discriminating. Thus the highest peak oxygen consumption might be expected in a patient with normal right ventricular long axis excursion and peak shortening rate and a dominant transmitral A wave. Well over half $(65 \%)$ of the variance of peak oxygen consumption could be predicted on this basis.

The association between right ventricular function and exercise tolerance in these patients with left ventricular disease confirms previous studies in which right ventricular ejection fraction was determined by nuclear angiography. However, right ventricular long axis function is very simple to measure by echo, and can be repeatedly estimated without radiation exposure. The finding of disease in the right coronary artery in most of the coronary artery disease group, with total occlusion in five, suggests that the depressed long axis excursion was secondary to intrinsic right ventricular disease rather than to increased pulmonary artery pressure causing abnormal loading. In addition, the use of cardiopulmonary bypass necessary for coronary artery bypass grafting independently depresses right ventricular long axis function. ${ }^{15}$

Patients with left ventricular dilatation operating at a fixed stroke volume depend on heart rate to increase cardiac output with exercise. In our patients peak heart rate on exercise correlated inversely with duration of mitral regurgitation at rest. Prolonged mitral regurgitation limits the time available for left ventricular filling; thus as heart rate increases with exercise, the time available for forward mitral flow falls and may reach a value insufficient to sustain cardiac output. ${ }^{16}$ In these patients with mild "functional" mitral regurgitation the apparent chronotropic incompetence due to prolonged mitral regurgitation may contribute to their limitation in maximum exercise capacity.

Patients with impaired left ventricular function have an abnormal ventilatory response to exercise - the more impaired their exercise tolerance, the greater their $\dot{\mathrm{V}} / \dot{\mathrm{V}}_{\mathrm{CO}_{2}}$ slope. In our patients, the relation between $\mathrm{VE} / \mathrm{V}_{\mathrm{CO}}$ slope and $\mathrm{M}$ mode isovolumic relaxation time was suggestive of this. Values were normal in four patients, while in the remainder there was strong negative correlation between isovolumic relaxation time and $\dot{\mathrm{VE}} / \dot{\mathrm{V}}_{\mathrm{CO}}$ slope, such that when the isovolumic relaxation time was $20 \mathrm{~ms}$ or less, the ventilation pattern was always abnormal. We were unable to identify a factor that reliably predicted which patients with a normal isovolumic relaxation time had a normal ventilatory response to exercise (falling on the right hand side of the majority illustrated in fig 2), but those with a normal response tended to be younger and to exercise for longer. Isovolumic relaxation time, like 
many other diastolic measurements, depends both on the presence of ventricular disease and on left atrial pressure. A normal value may thus occur in a patient in whom relaxation is indeed normal, or when prolonged relaxation is counterbalanced by increased left atrial pressure. The distribution in fig 2 would be compatible with these dual determinants.

Other factors are also involved in determining exercise tolerance in patients with severe heart disease-in particular the occurrence of peripheral changes in skeletal muscle and blood flow together with loss of physical fitness, which contribute to the impaired exercise tolerance seen in patients with heart disease. ${ }^{17}{ }^{18}$ In addition, it is very likely that changes in cardiac function occur with exercise that are not apparent at rest (for example, the localised impaired contraction that occurs in classic angina or the development of ischaemic mitral regurgitation on exercise). Nevertheless the greater part of the variance in exercise tolerance could be predicted by a simple noninvasive test that is routinely and repeatedly performed in these patients.

LIMITATIONS OF THE STUDY

We studied relatively few patients with advanced disease. The study might thus usefully be extended to determine if the same relations apply over a greater range of left ventricular dimensions and function. Most patients and controls had performed several previous cardiopulmonary exercise tests in our laboratory as part of other studies, so a major effect of any learning curve on exercise times and peak oxygen consumption is unlikely. In addition, inclusion in a trial of exercise training probably motivated them to perform better on exercise testing than a randomly selected group of subjects. The patients in this series constituted a "learning set"; a further series of patients needs to be studied in order to see if the specific relations seen in this set apply prospectively.

CONCLUSION

In patients in whom the left ventricle is dilated and poorly functioning, more than half the variance in peak oxygen consumption and ventilatory response to exercise can be predicted from factors measured on Doppler echocardiography at rest. We suggest that these correla- tions might form the basis of interactive studies designed to investigate the mechanisms of the abnormal exercise performance seen in these patients.

KMW-P is funded by a British Heart Foundation Junior Fellowship (London, UK), MYH by the Brompton special cardiac fund (London, UK), and AJSC is supported by the Viscount RoystonTrust.

1 Lipkin DP, Perrins J, Poole-Wilson PA. Respiratory gas exchange in the assessment of patients with impaired venricular function. Br Heart f 1985:54:321-8.

2 Franciosa JA, Park M, Levine TB. Lack of correlation between exercise capacity and indexes of resting left ventricular performance in heart failure. $\mathrm{Am} \mathrm{f}$ Cardiol 1981;47:33-9.

3 Higginbotham MB, Morris KG, Conn EH, et al. Determinants of variable exercise performance among patients with severe left ventricular dysfunction. Am $\mathcal{F}$ Cardiol 1983;51: $52-60$.

4 Lipkin DP, Canepa-Anson R, Stephens MR, et al. Factors determining symptoms in heart failure: comparison of fast and slow exercise tests. Br Heart 7 1986;55:439-45.

5 Baker BJ, Wilen MM, Boyd CM, et al. Relation of right ventricular ejection fraction to exercise capacity in chronic left tricular ejection fraction to exercise capacity in chro

6 Polak JF, Holman BL, Wynne J, et al. Right ventricular ejection fraction: an indicator of increased mortality in patients with congestive heart failure associated with coronary with congestive heart fallure associated with $\mathrm{Am}$ Coll Cardiol 1983;2:217-24.

7 Mancini D, Eisen H, Kussmaul W, et al. Value of peak exercise oxygen consumption for optimal timing of cardiac transplantation in ambulatory patients with heart failure. Circulation 1991;83:778-86.

8 Henein MY, Cailes J, O'Sullivan C, et al. Abnormal ventricular long-axis function in systemic sclerosis. Chest 1995;108:1533-40.

9 Upton MT, Gibson DG. The study of left ventricular function from digitized echocardiograms. Prog Cardiovasc Dis 1978;20:359-84

10 Bruce RA, Blackman JR, Jones JW. Exercise testing in adult normal subjects and cardiac patients. Pediatrics 1963;32: $742-55$

11 Packer M. Abnormalities of diastolic function as a potential cause of exercise intolerance in chronic heart failure. Circulation 1990;81(suppl III):III-78-86.

12 Franciosa JA, Leddy CL, Wilen M, et al. Relation between hemodynamic and ventilatory responses in determining exercise capacity in severe congestive heart failure. $A m \mathcal{F}$ Cardiol 1984;53:127-34.

13 Vanoverschelde J-LJ, Raphael DA, Robert AR, et al. Left ventricular filling in dilated cardiomyopathy: relation to functional class and hemodynamics. F $\mathrm{Am}$ Coll Cardiol 1990;15:1288-95.

14 Lewis BS, Emmott SN, Smyllie J, et al, and the DEFIANT Study Group. Left ventricular systolic and diastolic function, and exercise capacity six to eight weeks after acute myocardial infarction. Am f Cardiol 1993;72:149-53.

15 Koh TW, Henein MY, Gibson DG, et al. Persistent right ventricular dysfunction following coronary artery bypass ventricular dysfunction following coronary arte
grafting [abstract]. Heart 1997;77 (suppl 1):P40.

$16 \mathrm{Ng} \mathrm{KSK}$, Gibson DG. Impairment of diastolic function by hortened filling period in severe left ventricular disease. $\mathrm{Br}$ Heart f 1989;62:246-52.

17 Hambrecht R, Niebauer J, Fiehn E, et al. Physical training in patients with stable chronic heart failure: effects on cardiorespiratory fitness and ultrastructural abnormalities of leg muscles. F Am Coll Cardiol 1995;25:1239-49.

18 Coats AJS, Adamopoulos S, Meyer TE, et al. Effects of physical training in chronic heart failure. Lancet 1990;335: $63-6$ 